\title{
HEMANGIOMAS DE LA VULVA Y DE LA VAGINA
}

\author{
Doctor Enrique Darnalt Restrepo
}

Del Departamento de Ginecología del Instituto Nacional de Cancerología

\section{REVISION DE LA LITERATURA}

Presentación de dos casos de Hemangiomas vulvar y uno de Hemangioma vaginal (1).

Los hemangiomas son tumores benignos de los vasos sanguineos (1) y de acuerdo con su estructura y apariencia se clasifican en arteriales, capilares, venosos o cavernosos (2). La mayo_ ría de las veces son asintomáticos y su tamaño es variable tendiendo a ser pequeños. Son tumores levantados, papulosos o planos, de color violáceo, de contornos más bien netos y pueden aparecer zonas blanquecinas al ejercer presión sobre ellos. Se presentan más frecuentemente en los niños y pueden ser únicos o múltiples, es decir, localizados en varias partes del cuerpo. Generalmente congénitos. Según Anderson el verdadero hemangioma es congénito en su origen o pueden ser adquiridos los pequeños angiomas capilares. Histológicamente su apariencia es como un nido de vasos sanguíneos de diversos tamaños sostenidos por una capa simple de células endoteliales que pueden contener o no eritrocitos. El tejido conectivo o estroma es de una densidad más o menos variable (3).

Los tumores vaseulares de los órganos genitales femeninos no son frecuentes y tan solo algunos casos han sido publicados, hecho que está demostrado en la revisión de la literatura que da la impresión que sean escasos o que por el tamaño generalmente pequeño y la falta de síntomas pueden pasar desapercibidos. En. tre nosotros Valencia Piedrahita en su especial y laborioso estudio sobre Ginecopatías tumorales en la niñez, revisa la literatura y presenta cinco casos de hemangiomas vulvares on una se-

(1) Historias Clínicas pertenecientes al Instituto Nal. de Cancerología. 
rie de 39 casos de neoplasia genitales, es decir, el $55.55 \%$ (4). Por otra parte, Gerbie, Hirsch y Greene en su artículo Tumores vasculares del tracto genital femenino, refieren cinco casos de he. mangiomas entre 34 neoplasmas benignos de la vulva descritos por Lovelady, McDonald y Waugh. Holstein y Billich informan de un caso de hiperplasia vascular de la vagina con características angiomatosas (5). Alfieri, De Lima y Tiscornia y Ayllon $(6,7,8)$ refieren algunos casos. Otras localizaciones menos frecuentes de los hemangiomas y que han sido relatados son el cervix, el cuerpo uterino, los ligamentos redondos, las trompas y los ovarios (3).

Por las razones anteriormente expuestas nos hemos permitido publicar tres casos de hemangiomas, dos vulvares y uno vaginal.

Los casos de hemangioma vulvar se presentaron en niñas de cuatro y tres meses de edad respectivamente y por información de las madres fueron observadas desde el nacimiento, es decir, de tipo congénito. El caso del hemangioma vaginal raro e interesante por su aparición, evolución y apreciación clínica se presentó en una mujer multípara de treinta y cinco años, con sintomatología clínica de hemorragias vaginales de 20 meses de evolución y una lesión vaginal sospechosa de ser o un carcinoma primitivo o una endometriosis externa y cuyo diagnóstico defini-tivo lo decidió el estudio anatomo-patológico de la lesión extirpada.

El tratamiento de los hemangiomas es quirúrgico. La aplicación de Radium o de Rayos $\mathbf{X}$ no dio buen resultado en estos casos, ya que en uno de los vuivares no regresó con aplicación de Radium y el vaginal que fue tratado con Roengenterapia de contacto tuvo necesidad de ser extirpado de nuevo quirúrgicamente. El pronóstico es bueno.

Caso número 1.-Historia clínica número 38057. Noviembre 9/55. Niña de cuatro meses de edad. Desde el nacimiento observaron la presencia de lesiones violáceas en el gran labio vulvar derecho, en el costado derecho a nivel de la décima costilla y en la mano derecha hacia la raíz del dedo indice y pulgar.

Al examen presenta en el gran labio derecho de la vulva tumor de color violáceo, de 2 centímetros de diámetro, móvil, no adherentes a los planos profundos. La piel está sana. En la región costal derecha sobre la línea axilar media existe una tumoración blanda, de un diámetro aproximadamente de 3 centíme- 


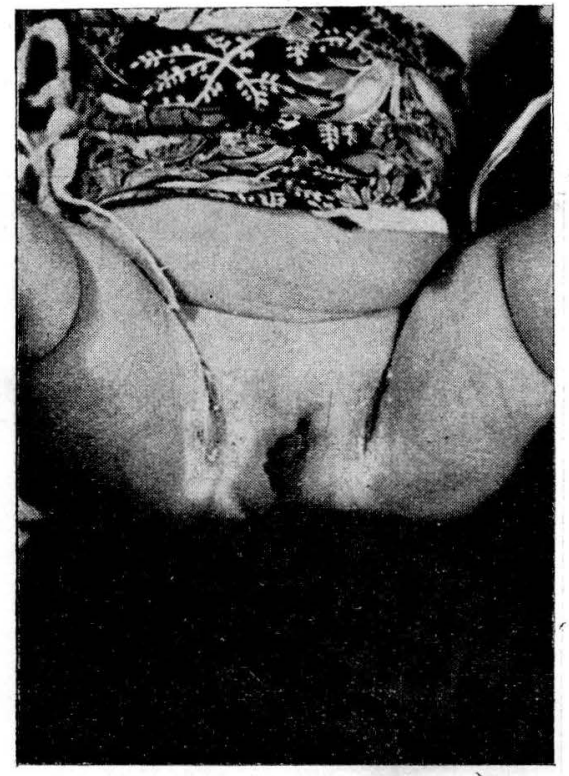

Caso número 1 Hemangioma de la vulva

tros. También violáceo. En la mano derecha, dorso y dedos indice y pulgar existen manchas angiomatosas. En diciembre de 1955 practicaron tratamiento de Radium superficial sobre la lesión vulvar con placa de plomo y 8 agujas de $1 . \mathrm{mgm}$., con una dosis total de 64 mgrm. y en vista de no haber obtenido buenos resultados se resuelve la extirpación quirúrgica.

Diagnóstico anatomo-patológico.-La pieza consiste en un fragmento de piel de color rosado azuloso de 1.5 centimetros de diámetro. Al corte se aprecian tejidos de aspecto fibroso y escasa cantidad de tejido adiposo.

Las preparaciones muestran un hemangioma. Existe proliferación endotelial y formación de vasos sanguíneos, la mayoría pequeños. Contienen glóbulos rojos. No hay malignidad. Diagnóstico: Hemangioma de la vulva.

Examen de control: septiembre 13/57. Los angiomas de la mano han desaparecido en forma espontánea casi totalmente. En la vulva se ven muy pequeñas manchas rojas superficiales, pero el elemento primitivo aparece curado. El Hemangioma del hemitórax derecho no ha crecido. 
Caso número 2.-Historia clínica número 43331. Septiembre 16/57. Niña de tres meses de edad. Desde el nacimiento notaron tumoraciones y manchas violáceas en el antebrazo izquierdo, labio superior y en la vulva.

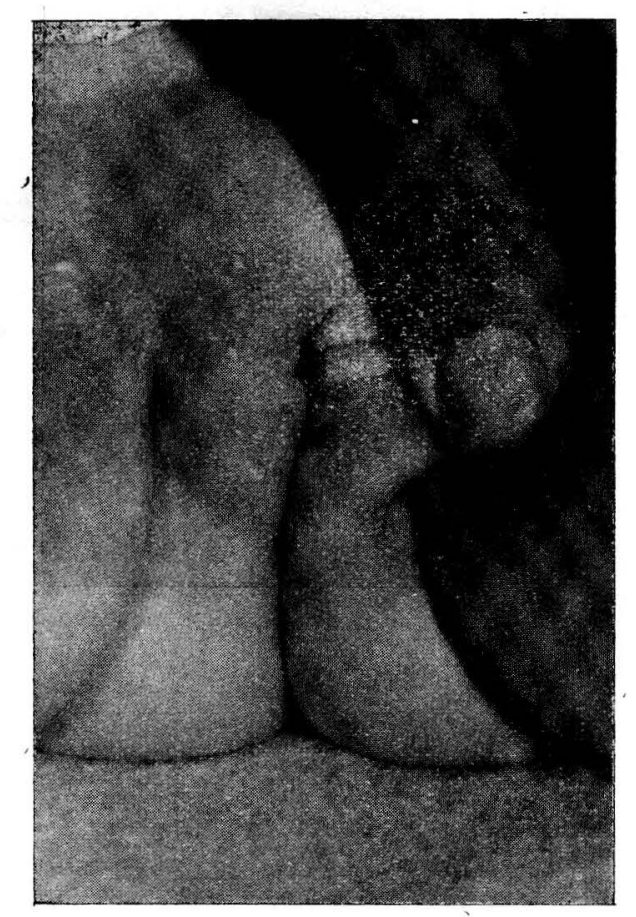

Caso número 2 de hemangioma de la vulva

Examen: Hemangiomas de color violáceo. El más grande está localizado en el borde radial del tercio inferior del antebrazo izquierdo, es levantado en relieve sobre la piel. En la mitad derecha del labio superior sobre el lado bucal se ve una mancha plana de $5 \mathrm{~mm}$. de diámetro, de color resado. En la vulva sobre el labio mayor izquierdo, tercio posterior, elemento macular de $5 \mathrm{~mm}$. de diámetro, de co'or rojo, ligeramente en relieve.

Diagnóstico: Hemangioma del labio superior, antebrazo y vulva. Congénitos.

La paciente es enviada a la sección de Radioterapia para tratamiento del hemangioma del antebrazo. Los de la vulva y labio superior se dejan en observación por ser bastante peque. ños y pueden regresar jin tratamiento. 
Caso número 3.-Historia clínica número 37818. Octubre 10 de 1955. Mujer de treinta y cinco años. Multípara. Consulta por hemorragias vaginales acíclicas desde febrero de 1954. En septiembre del mismo año practicaron Anexo-histerectomía total por absceso pélvico. En febrero de 1955 colecistectomía por calculosis. Luego después de la anexo-histerectom'a las hemorragias vaginales continuaron y al examinarla observaron una erosión en la cara anterior de la vagina que fue electro-cauterizada; posteriormente fue tratada con estrógenos que aumentaron la cantidad de las hemorragias. Un estudio de citología vaginal mos. tró abundantes células carcítomatosas y biopsias de la lesión vaginal dieron como resultado: Fibrosis y ulceración.

Remitida al Instituto Nacional de Cancerología se encuentra al examen: abdomen globuloso, obeso. Cicatrices quirúrgicas en buenas condiciones. No se palpan masas. Genitales externos multíparos, relajados.

T. V.: La cúpula vaginal se encuentra cicatrizada. Sobre la pared vaginal anterior y lateral derecha se tacta una zona rugosa sin bases duras, cuyo diámetro aproximado es de 1 centímetro.

T. R.: Parametrios tensos.

Especulum: se observa hacia la cúpula, pared anterior, lado derecho, una zona destruída, sin bases duras, sangra con alguna facilidad y de un diámetro aproximado de 1 centímetro. No da la impresión clínica de un carcinoma, parece tratarse de una Endometriosis externa.

Se tomaron biopsias y frotis para citología vaginal, cuyos resultados fueron: proceso inflamatorio no específico. En las placas estudiadas no se vieron células tumorales.

Se resuelve extirpar la lesión quirúrgicamente y su resultado histológico es el siguiente: los cortes muestran mucosa vaginal y tejido conjuntivo subyacente. El epitelio está ulcerado en grandes extensiones y reemplazado por tejido de granulación no específico, hemorrágico en la superficie. En un sitio llama la aten. eión la presencia de numerosos canales vasculares, algunos de pared bastante gruesa. No se ven focos de endometriosis ni malignidad.

Diagnóstico anatomo-patológico: Hemangioma cavernoso con inflamación crónica.

Posteriormente informó la paciente que dos meses después de la intervención había presentado pequeñas hemorragias vaginales en forma casi permanente. Al examen se encontraron 
erosiones localizadas en el sitio primitivo de la lesión vaginal y se resolvió la aplicación de Rayos X intracavitaria de contacto, dando una dosis total de $2.950 \mathrm{r}$.

Como la enferma continuara presentando manchas hemáticas, practicaron nueva escisión quirúrgica de la lesión vaginal. El resultado anatomo-patológico es el siguiente:

Las alteraciones del tejido parecen corresponder a procesos distintos y es claro además cuentan las modificaciones provocadas naturalmente por los Rayos X. Existe un claro factor angiomatoso, pero está reducido a una mínima parte do la zona que comprende la lesión. Por esto nos da la impresión que los tras. tornos no son sino en pequeña parte imputables a él. Tampoco se trata de un tumor propiamente dicho en el sentido anatomohistológico, sino de zonas difusas angiomatosas.

Diagnóstico Histo-patológico: Hemangioma capilar y proceso granulomatoso ulcerado.

\section{Sumario}

Se presentan dos casos de Hemangioma vulvar y un caso de Hemangioma vaginal.

Se revisa gran parte de la literatura sobre Hemangiomas del aparato genital femenino.

\section{BIBLIOGRAFIA}

1. EWING, J.-“Angiomas". Neoplastic Descases. Philadelphia, Saunders, 1940.

2. WATSON, W. L., y McCARThY, W. D. Blcod and Lymph vessel tumors. Surg., Gynec. \& Obst. 71: 569, 1940.

3. BRANDFASS, R. T. y SCHEFFEY, L. C. Hemangiomas of the Uterine Cervix. Obst. \& Gynec. Vol. 6. № 5. 1955.

4. VALENCIA PIEDRAHITA GUILLERMO. Ginecopatías tumorales en la infancia. Rev. Col. Pediatría y Puericultura. No 3. Febr. 1955.

5. GERBIE, A, B., HIRSCH, M. R., y GREENE, R. R. Vascular Tumors of the Female Genital Tract. Obst. \& Gynec. Vol. 6. № 5. 1955.

6. ALFIERI, P. Angioma de la vulva. Clin. Ostet. 48: 145. 1946.

7. TISCORNIA BLAUS, A. y AYLLON, J. A. Hemangiomas of the external female genitals. Semana Med. 3: 1.419. 1936.

8. DE LIMA, O. A., Jr. Hemangioma de la vulva. Rev. Ginec. e Obst. 1: 47. 1952 . 